\title{
Quantum corrections in string compactifications on $\mathrm{SU}(3)$ structure geometries
}

\author{
Mariana Graña, ${ }^{a}$ Jan Louis, ${ }^{b, c}$ Ulrich Theis $^{d}$ and Daniel Waldram ${ }^{e}$ \\ a Institut de Physique Théorique, CEA/Saclay \\ 91191 Gif-sur-Yvette Cedex, France \\ ${ }^{b}$ Fachbereich Physik der Universität Hamburg \\ Luruper Chaussee 149, 22761 Hamburg, Germany \\ ${ }^{c}$ Zentrum für Mathematische Physik, Universität Hamburg \\ Bundesstrasse 55, 20146 Hamburg, Germany \\ ${ }^{d}$ Riemann Center for Geometry and Physics, Leibniz Universität Hannover, \\ Appelstrasse 2, 30167 Hannover, Germany \\ e Department of Physics, Imperial College London \\ London, SWr 2AZ, U.K. \\ E-mail: mariana.grana@cea.fr, jan.louis@desy.de, \\ ulrich.theis@riemann.uni-hannover.de, d. waldram@imperial.ac.uk
}

ABSTRACT: We investigate quantum corrections to the classical four-dimensional lowenergy effective action of type II string theory compactified on $\mathrm{SU}(3)$ structure geometries. Various methods previously developed for Calabi-Yau compactifications are adopted to constrain - under some simple assumptions about the low-energy degrees of freedom - the leading perturbative corrections to the moduli space metrics in both $\alpha^{\prime}$ and the string coupling constant. We find that they can be parametrized by a moduli dependent function in the hypermultiplet sector and a constant in the vector multiplet sector. We argue that under specific additional assumption they take - in complete analogy to the Calabi-Yau case - a universal form which depends only on the Euler characteristic of the six-dimensional compact space.

KEYWORDS: Supersymmetric Effective Theories, Flux compactifications, Superstring Vacua ARXiv EPRINT: 1406.0958 


\section{Contents}

1 Introduction 1

2 Preliminaries $\quad 2$

$2.1 \mathcal{N}=2$ supersymmetry in $M_{1,3}$

$2.2 \mathrm{SU}(3)$ compactifications 4

3 Perturbative $\alpha^{\prime}$ and $g_{s}$ corrections $\quad 6$

$\begin{array}{lll}3.1 & \text { Constraints on the metric moduli } & 7\end{array}$

$3.1 .1 \alpha^{\prime}$ corrections $\quad 8$

$\begin{array}{lll}3.1 .2 & g_{s} \text { corrections } & 10\end{array}$

$\begin{array}{lll}3.2 & \text { Perturbative } \alpha^{\prime} \text { corrections } & 11\end{array}$

$\begin{array}{lll}3.3 & \text { Perturbative } g_{s} \text { corrections } & 14\end{array}$

4 Summary and outlook $\quad 16$

\section{Introduction}

In this paper we consider type II string theory in backgrounds $M_{1,3} \times X_{6}$, where $M_{1,3}$ is a four-dimensional $(d=4)$ Lorentzian space-time and $X_{6}$ is a compact six-dimensional manifold. Demanding that the effective theory in $M_{1,3}$ admit eight unbroken supercharges $(\mathcal{N}=2$ in $d=4)$ constrains $X_{6}$ to be a manifold with $\mathrm{SU}(3)$ structure [1-5]. ${ }^{1}$ Manifolds with SU(3) structure admit a globally defined nowhere-vanishing spinor and as a consequence the structure group of the tangent space is reduced, meaning that it can be patched using only an $\mathrm{SU}(3)$ subgroup of $\mathrm{SO}(6)$. If in addition the spinor is covariantly constant the Levi-Civita connection has SU(3) holonomy, and in this case $X_{6}$ is a Calabi-Yau manifold.

The $\mathcal{N}=2$ low-energy effective Lagrangian of such backgrounds has been computed in refs. $[4,5,14-23]$ at the string tree level and for "large" manifolds $X_{6}$ assuming a suitable Kaluza-Klein reduction. For Calabi-Yau manifolds this is straightforward in that the massless modes are in one-to-one correspondence with the cohomology of $X_{6}$, and the resulting $\mathcal{N}=2$ supergravity is ungauged with no potential [24-26]. In the generalized case the corresponding analysis is much harder and can only be performed if there is a suitable hierarchy of the low-energy modes. In this case the effective action is a gauged $\mathcal{N}=2$ supergravity with a potential which stabilizes (some of) the moduli.

The low-energy effective Lagrangian is corrected by higher-order $\alpha^{\prime}$ terms as well as by string loops $\left(g_{s}\right.$ corrections). Both types of corrections can be parametrized by a scalar field

\footnotetext{
${ }^{1}$ More generally, viewed as generalized geometries $[6-11], \mathcal{N}=2$ supersymmetry requires an $\mathrm{SU}(3) \times$ $\mathrm{SU}(3)$ structure on the generalized tangent space [12-15] or even more generally an $\mathrm{SU}(6)$ structure on the exceptional generalized tangent space [16].
} 
(or a modulus) in the low-energy effective action. Higher-order $\alpha^{\prime}$ corrections arise when the size of the manifold is comparable to the string length and thus they are controlled by the volume modulus of $X_{6}$. String loop corrections on the other hand are counted by $g_{s}$ with the dilaton being the corresponding modulus. For Calabi-Yau compactifications both types of corrections have been partially computed, and we review some of these results in the following. However, for generalized compactifications little is known about these corrections primarily due to the fact that they have no direct worldsheet description.

The purpose of this paper is to investigate what can be said about $\alpha^{\prime}$ and $g_{s}$ corrections in the specific case of $\mathrm{SU}(3)$ structure compactifications. We will not focus on a particular background but rather attempt to constrain the generic form of the possible corrections, under some simple assumptions. We also do not consider any non-trivial NSNS or RR fluxes, or more general $\mathrm{SU}(3) \times \mathrm{SU}(3)$ structures (see footnote 1 ).

Using simple extensions of the arguments employed for Calabi-Yau compactifications in [27-32] we find - given there is an expansion in terms of a finite set of low-energy fields - that the $\mathcal{N}=2$ supersymmetry strongly constrains the leading corrections to the kinetic terms. In the hypermultiplet sector a non-renormalization theorem holds and the metric is perturbatively corrected only at one-loop by a moduli dependent function. In the type IIA vector multiplet sector we find $\alpha^{\prime}$ corrections which in a large volume expansion correct the holomorphic prepotential by a constant term at leading order. In type IIB the vector multiplet sector on the other hand is not corrected at all. With the additional assumption of a putative mirror symmetry we are able to argue that all corrections are proportional to the Euler characteristic of $X_{6}$ exactly as in Calabi-Yau compactifications.

Generically, SU(3) structure compactifications admit fewer Peccei-Quinn (PQ) symmetries than Calabi-Yau compactifications, since some of the corresponding moduli acquire a mass from the gauging of the $\mathcal{N}=2$ supergravity. Remarkably, we find that the leading kinetic energy corrections still have the full set of PQ symmetries, even under shifts of the massive moduli.

The paper is organized as follows: in section 2 we review some basic facts about $\mathcal{N}=2$ supergravity and $\mathrm{SU}(3)$ structure compactifications. We also summarize our assumptions about the moduli space of low-energy fields. Section 3 then discusses the form of the leading corrections to the metrics on these moduli spaces: the prepotential for the vector multiplet fields, and the quaternionic-Kähler metric for the hypermultiplet fields. We first discuss the constraints on the metric of the geometric moduli that arise from dimensionally reducing the known ten-dimensional $R^{4}$ correction terms. We then turn to the full vector and hypermultiplet moduli space metrics, first for the $\alpha^{\prime}$ corrections and then for the $g_{s}$ corrections. Section 4 summarizes our results and contains some concluding remarks.

\section{Preliminaries}

\section{$2.1 \mathcal{N}=2$ supersymmetry in $M_{1,3}$}

In order to discuss the $\alpha^{\prime}$ and $g_{s}$ corrections we need to briefly assemble some facts about $\mathcal{N}=2$ supergravity (for a review see e.g. [33]). A generic spectrum contains a gravita- 
tional multiplet, $n_{\mathrm{v}}$ vector multiplets and $n_{\mathrm{h}}$ hypermultiplets. ${ }^{2}$ The gravitational multiplet contains the spacetime metric, two gravitini and the graviphoton $A_{\mu}^{0}$. A vector multiplet contains a vector $A_{\mu}$, two gaugini and a complex scalar $t$. Finally, a hypermultiplet contains two hyperini and four real scalars $\left(q^{1}, q^{2}, q^{3}, q^{4}\right)$.

The $\mathcal{N}=2$ supersymmetry enforces the scalar field space $M$ locally to be a product

$$
M=M_{\mathrm{v}} \times M_{\mathrm{h}},
$$

where $M_{\mathrm{v}}$ is a $2 n_{\mathrm{v}}$-dimensional manifold spanned by the complex scalars $t^{i}, i=1, \ldots, n_{\mathrm{v}}$, contained in $n_{\mathrm{v}}$ vector multiplets, while $M_{\mathrm{h}}$ is a $4 n_{\mathrm{h}}$-dimensional manifold spanned by the real scalars $q^{u}, u=1, \ldots, 4 n_{\mathrm{h}}$, in $n_{\mathrm{h}}$ hypermultiplets. Thus their sigma-model Lagrangian is of the form

$$
\mathcal{L}=g_{i \bar{\jmath}}(t, \bar{t}) D_{\mu} t^{i} D^{\mu} \bar{t}^{\bar{\jmath}}+h_{u v}(q) D_{\mu} q^{u} D^{\mu} q^{v}-V(t, \bar{t}, q), \quad \mu=0, \ldots, 3,
$$

where $g_{i \bar{j}}$ is the metric on $M_{\mathrm{v}}$ while $h_{u v}$ is the metric on $M_{\mathrm{h}}$. In general, isometries on $M_{\mathrm{v}}$ and $M_{\mathrm{h}}$ can be gauged, so that $D$ is an appropriate covariant derivative which includes the couplings of the charged scalars to the vector bosons. Only if the theory is gauged can it admit a non-trivial potential $V$, which furthermore is completely determined by the choice of gauging.

Supersymmetry further constrains $M_{\mathrm{v}}$ to be a special-Kähler manifold, so that the metric can be written as [34, 35]

$$
g_{i \bar{\jmath}}=\partial_{i} \partial_{\bar{\jmath}} K^{\mathrm{v}} \quad \text { with } \quad K^{\mathrm{v}}=-\ln \mathrm{i}\left(\bar{X}^{I} \mathcal{F}_{I}-X^{I} \overline{\mathcal{F}}_{I}\right) .
$$

Both $X^{I}(t)$ and $\mathcal{F}_{I}(t), I=0,1, \ldots, n_{\mathrm{v}}$, are holomorphic functions of the scalars $t^{i}$, and $\mathcal{F}_{I}=\partial \mathcal{F} / \partial X^{I}$ is the derivative of a holomorphic prepotential $\mathcal{F}(X)$ homogeneous of degree two. Furthermore, it is possible to adopt a system of 'special coordinates' in which $X^{I}=$ (i, $t^{i}$ ) (see e.g. [35] for further details).

$M_{\mathrm{h}}$ is similarly constrained to be a quaternionic-Kähler manifold, which means its holonomy group is of the form $\mathrm{Sp}(1) \times \mathrm{H}$ with $H \subset \mathrm{Sp}\left(\mathrm{n}_{\mathrm{h}}\right)$ [36, 37]. There is a special class of quaternionic-Kähler manifolds which arise in string tree-level effective actions known as 'special quaternionic-Kähler manifolds' $M_{\mathrm{SQK}}^{4 n_{\mathrm{h}}}$. These manifolds can be viewed as a $2 n_{\mathrm{h}}$ dimensional torus fibred over a special-Kähler base, of the form $[\mathrm{SU}(1,1) / \mathrm{U}(1)] \times \mathrm{M}_{\mathrm{SK}}^{2 \mathrm{n}_{\mathrm{h}}-2}$, where $M_{\mathrm{SK}}^{2 n_{\mathrm{h}}-2}$ is a special-Kähler manifold of dimension $2 n_{\mathrm{h}}-2$. This relation between special-Kähler and quaternionic Kähler manifolds is known as the 'c-map' [38]

$$
\frac{\mathrm{SU}(1,1)}{\mathrm{U}(1)} \times M_{\mathrm{SK}}^{2 n_{\mathrm{h}}-2} \mapsto M_{\mathrm{SQK}}^{4 n_{\mathrm{h}}} .
$$

The metric on $M_{\mathrm{SQK}}^{4 n}$ takes an explicit form known as the Ferrara-Sabharwal metric, which reads $[39,40]$

$$
\begin{aligned}
\mathcal{L}= & \left(\partial_{\mu} \phi\right)^{2}+\mathrm{e}^{4 \phi}\left(\partial_{\mu} \sigma-\xi^{I} \partial_{\mu} \tilde{\xi}_{I}\right)^{2}+g_{a \bar{b}} \partial_{\mu} z^{a} \partial^{\mu} \bar{z}^{\bar{b}} \\
& -\mathrm{e}^{2 \phi}(\mathcal{N}+\overline{\mathcal{N}})^{I J}\left(\partial_{\mu} \tilde{\xi}_{I}-2 \mathrm{i} \mathcal{N}_{I K} \partial_{\mu} \xi^{K}\right)\left(\partial^{\mu} \tilde{\xi}_{J}+2 \mathrm{i} \overline{\mathcal{N}}_{J L} \partial^{\mu} \xi^{L}\right),
\end{aligned}
$$

\footnotetext{
${ }^{2}$ Tensor multiplets are dual to hypermultiplets if they are massless and to vector multiplets if they are massive.
} 
where $\phi$ and $\sigma$ span the $\mathrm{SU}(1,1) / \mathrm{U}(1)$ factor of $(2.4), \xi^{I}$ and $\tilde{\xi}_{I}$ are real coordinates of the torus fibre, and $z^{a}$ are complex coordinates on the special Kähler base $M_{\mathrm{SK}}^{2 n_{\mathrm{h}}-2}{ }^{3}$ The metric $g_{a \bar{b}}(z, \bar{z})$ on $M_{\mathrm{SK}}^{2 n_{\mathrm{h}}-2}$ satisfies (2.3) and thus can be characterized by a holomorphic prepotential $\mathcal{G}(z) .{ }^{4}$ The couplings $\mathcal{N}_{I J}(z, \bar{z})$ are given in terms of $\mathcal{G}$ as

$$
\mathcal{N}_{I J}=-\mathrm{i} \overline{\mathcal{G}}_{I J}+2 \frac{\operatorname{Im} \mathcal{G}_{I K} z^{K} \operatorname{Im} \mathcal{G}_{J L} z^{L}}{\operatorname{Im} \mathcal{G}_{M N} z^{M} z^{N}}
$$

with $z^{I}=\left(\mathrm{i}, z^{a}\right)$. In type II string theory reduced on, in particular, a Calabi-Yau manifold, $\phi$ and $\sigma$ are identified with the four-dimensional dilaton and universal axion, respectively, while $\xi^{I}$ and $\tilde{\xi}_{I}$ are identified with four-dimensional scalar fields arising from the RR sector.

\section{$2.2 \mathrm{SU}(3)$ compactifications}

Let us now briefly discuss the notion of an $\mathrm{SU}(3)$ compactification. Recall first the moduli that appear when the compactification space $X_{6}$ is a Calabi-Yau manifold. One finds that the scalar fields in vector- and hypermultiplets arise from $h^{(1,1)}$ deformations of the complexified Kähler form $J+\mathrm{i} B$ and from $h^{(1,2)}$ deformations of the complex structure, or equivalently deformations of the holomorphic 3 -form $\Omega$. Since these moduli can be varied independently, their moduli space is locally a product

$$
M\left(X_{6}\right)=M_{J} \times M_{\Omega}
$$

with each component being a special-Kähler manifold. Their respective Kähler potentials are given, prior to $\alpha^{\prime}$ and string loop corrections, by [24-26]

$$
K_{J}=-\ln \int_{X_{6}} J \wedge J \wedge J, \quad K_{\Omega}=-\ln \mathrm{i} \int_{X_{6}} \Omega \wedge \bar{\Omega} .
$$

In type IIA compactifications one has $M_{J}=M_{\mathrm{v}}$ while $M_{\Omega}=M_{\mathrm{SK}}^{2 h^{(1,2)}}$ is the specialKähler base in the c-map (2.4). The full space $M_{\mathrm{h}}$ also includes the dilaton and axion plus $2 h^{(1,2)}+2$ scalars from the RR sector which parameterize the torus fibre. In type IIB compactifications the situation is reversed and one has $M_{\Omega}=M_{\mathrm{v}}$ while $M_{J}=M_{\mathrm{SK}}^{2 h^{(1,1)}}$ is the special-Kähler base. The dilaton and axion plus $2 h^{(1,1)}+2$ scalars from the RR sector again complete $M_{\mathrm{h}}$.

In this paper we will be considering the weaker case where we only assume that $X_{6}$ admits an SU(3) structure [1-3]. This means we can still find a globally defined fundamental 2 -form $J$ and an almost complex structure with a corresponding globally defined complex $(3,0)$-form $\Omega$. However, they are now no longer closed, so that

$$
\mathrm{d} J \neq 0, \quad \mathrm{~d} \Omega \neq 0,
$$

\footnotetext{
${ }^{3} \operatorname{In}(2.5)$ we display the ungauged metric. If some of its isometries are gauged the corresponding ordinary derivatives are replaced by appropriate covariant derivatives.

${ }^{4}$ For type IIA, we denote the coordinates and prepotential of $M_{\mathrm{SK}}^{2 n_{\mathrm{h}}-2}$ in the hyper-sector by $z^{a}$ and $\mathcal{G}$, respectively, in order to distinguish it from the coordinates $t^{i}$ and prepotential $\mathcal{F}$ in the vector multiplet sector. For type IIB the rôles of $t^{i}$ and $z^{a}$ and $\mathcal{F}$ and $\mathcal{G}$ will be reversed.
} 
and one says that the intrinsic torsion of the $\mathrm{SU}(3)$ structure on $X_{6}$ is non-zero. This lack of integrability means that the space of generic $\mathrm{SU}(3)$ structures on $X_{6}$ is infinite-dimensional, though it nonetheless still decomposes as a product of special-Kähler manifolds [14, 15], with Kähler potentials given by (2.8). In the corresponding 'low-energy' effective action the intrinsic torsion plays the role of gauge charges and/or mass terms. As a consequence, the four-dimensional supergravity is gauged.

For a realistic theory, we would like to identify a finite-dimensional subspace of SU(3) structures, as we had for the Calabi-Yau case. This should correspond to identifying some light "moduli" fields in the Kaluza-Klein spectrum of all possible deformations of $J$ and $\Omega$. In this paper we will simply assume such an expansion exists and then investigate the consequences for quantum corrections to the corresponding effective action. Our assumptions are (see $[4,5,14,15,18]$ for details):

1. There is a finite-dimensional subspace $M$ of $\mathrm{SU}(3)$ structures such that we have the expansion

$$
J+\mathrm{i} B=t^{i} \omega_{i}, \quad \Omega=z^{I} \alpha_{I}-\mathcal{G}_{I}(z) \beta^{I} .
$$

In contrast to the Calabi-Yau case, the lack of integrability (2.9) means that the basis two-forms $\omega_{i}$ and the dual four-forms $\tilde{\omega}^{i}$ as well as the three-forms $\alpha_{I}$ and $\beta^{I}$ are no longer necessarily harmonic; these forms constitute a closed set under the Hodge star and also under the exterior derivative, namely

$$
\begin{array}{rlrl}
\mathrm{d} \alpha_{I} & \sim e_{I i} \tilde{\omega}^{i}, & \mathrm{~d} \beta^{I} \sim p_{i}^{I} \tilde{\omega}^{i}, \\
\mathrm{~d} \omega_{i} & \sim p_{i}^{I} \alpha_{I}-e_{i I} \beta^{I}, & & \mathrm{~d} \tilde{\omega}^{i} \sim 0,
\end{array}
$$

where $e_{i I}$ and $p_{i}^{I}$ are constant matrices.

2. There are no additional light spin-3/2 fields other than the $\mathcal{N}=2$ gravitini (this corresponds to an absence of moduli of type $(1,0)$ with respect to the almost complex structure $[14,15])$.

3. There is an expansion around the large-volume limit. The moduli space manifestly includes the volume modulus $V$ since this is a rescaling of the real parts of $t^{i}$, here we in addition assume that the moduli are light compared with other Kaluza-Klein modes in the large-volume region.

This set-up can be viewed as the SU(3) structure analogue of a Calabi-Yau compactification with flux. The flux generates a potential for the Calabi-Yau moduli, but in the large volume limit this potential is generically small compared to the masses of the Kaluza-Klein modes. As a result the low-energy theory is just a gauged $\mathcal{N}=2$ supergravity theory for the moduli fields. Here we are requiring the existence of a family of SU(3) structures which are "close" to satisfying the Einstein equations, in the sense that the Ricci curvature is small compared to the Kaluza-Klein scale in the large volume limit.

The existence of an $\mathrm{SU}(3)$ structure implies that the effective field theory in four dimensions is $\mathcal{N}=2$ supersymmetric. Given our assumptions, the moduli space is again a product

$$
M=M_{J} \times M_{\Omega}
$$


of a moduli space $M_{J}$ of deformations of $J+\mathrm{i} B$ and of a moduli space $M_{\Omega}$ of deformations of $\Omega$, where the volume modulus $V$ is part of $M_{J}$. Furthermore, the expansion (2.10) is such that the special Kähler structures on the infinite dimensional space of all $\mathrm{SU}(3)$-structures restrict to special Kähler structures on each component $M_{J}$ and $M_{\Omega}$ with Kähler potentials again given by (2.8). In addition, prior to including $\alpha^{\prime}$ and string loop corrections, the lowenergy effective theory is a gauged $\mathcal{N}=2$ supergravity theory.

The RR potentials are assumed to admit an expansion in the same bases, namely $\alpha_{I}$ and $\beta^{I}$ in type IIA and $\omega_{i}, \tilde{\omega}^{i}$ and the volume form $\varepsilon$ in type IIB, so that

$$
\begin{aligned}
& C_{3}=\xi^{I} \alpha_{I}+\tilde{\xi}_{I} \beta^{I}, \\
& C_{0}=\xi^{0}, \quad C_{2}=\xi^{i} \omega_{i}, \quad C_{4}=\tilde{\xi}_{i} \tilde{\omega}^{i}, \quad C_{6}=\tilde{\xi}_{0} \varepsilon .
\end{aligned}
$$

In Calabi-Yau compactifications the metric on the quaternionic manifold $M_{\mathrm{h}}$ takes the Ferrara-Sabharwal form (2.5) to leading order. Related to the torus fibre the metric has $2 n_{\mathrm{h}}+1$ (perturbative) Peccei-Quinn shift symmetries which read

$$
\sigma \rightarrow \sigma+\gamma+c^{I} \tilde{\xi}_{I}, \quad \xi^{I} \rightarrow \xi^{I}+c^{I}, \quad \tilde{\xi}_{I} \rightarrow \tilde{\xi}_{I}+\tilde{c}_{I}, \quad \gamma, c^{I}, \tilde{c}_{I} \in \mathbb{R} .
$$

These symmetries arise from large gauge transformations of the type II $p$-form fields $C_{p}$, that is, shifting them by constant multiples of the (closed) basis forms $\omega_{i}$ etc. They are broken to discrete shift symmetries by non-perturbative space-time physics but nevertheless imply a perturbative non-renormalization theorem in that perturbative corrections of the hypermultiplets only occur at one-loop but not beyond [27-29, 31, 32].

By contrast, for $\mathrm{SU}(3)$ structures the basis forms are generically no longer harmonic, so in general the shift symmetries (2.14) are broken and the corresponding scalar fields are massive. Nonetheless, simple dimensional analysis shows that the leading-order calculation of the kinetic terms does not see the derivatives of the basis forms and so the metric on the hypermultiplet space still takes the Ferrara-Sabharwal form (2.5). At higher order in $\alpha^{\prime}$ and $g_{s}$ one would naively expect that corrections to the metric see the fact that the Peccei-Quinn symmetries are broken.

In summary, our assumptions imply that at leading order — just as in the Calabi-Yau case - in type IIA one has $M_{J}=M_{\mathrm{v}}$ while $M_{\Omega}$ is the base of a special quaternionic-Kähler manifold $M_{\mathrm{h}}$. In type IIB the situation is reversed and one has $M_{\Omega}=M_{\mathrm{v}}$ while $M_{J}$ is the base of $M_{\mathrm{h}}$. In both cases the special quaternionic manifold $M_{\mathrm{h}}$ includes the dilaton and axion.

\section{$3 \quad$ Perturbative $\alpha^{\prime}$ and $g_{s}$ corrections}

In this section we discuss the structure of the perturbative $\alpha^{\prime}$ and $g_{s}$ corrections to the moduli spaces metrics in the class of $\mathrm{SU}(3)$ structure compactifications defined above, focussing on the leading-order contributions. The key point is that they are strongly constrained by the $\mathcal{N}=2$ supersymmetry which by construction survives the compactification. In essence we find that several of the usual arguments that apply to Calabi-Yau compactifications go through in this case too. 


\begin{tabular}{|c|c|c|c|c|}
\hline corrections & $M_{\mathrm{v}}^{\text {IIA }}$ & $M_{\mathrm{h}}^{\mathrm{IIA}}$ & $M_{\mathrm{v}}^{\mathrm{IIB}}$ & $M_{\mathrm{h}}^{\mathrm{IIB}}$ \\
\hline$\alpha^{\prime}$ & yes & no & no & yes \\
\hline$g_{s}$ & no & yes & no & yes \\
\hline
\end{tabular}

Table 1. Structure of $\alpha^{\prime}$ and $g_{s}$ corrections in type IIA and type IIB.

First note that if the low energy spectrum contains no massive spin-3/2 multiplets, $\mathcal{N}=2$ supersymmetry enforces the split (2.1) into locally independent special-Kähler and quaternionic moduli spaces, which thus has to persist after including perturbative and nonperturbative $\alpha^{\prime}$ and $g_{s}$ corrections. The $\alpha^{\prime}$ corrections enter in an expansion in the inverse of the volume modulus $V^{-1}$, while the loop corrections are in powers of the four-dimensional dilaton $\mathrm{e}^{2 \phi}=V^{-1} \mathrm{e}^{2 \varphi}$. However, in both type II compactifications the dilaton is part of a hypermultiplet and thus the component $M_{\mathrm{h}}$ receives quantum corrections, whereas $M_{\mathrm{v}}$ is uncorrected and thus "exact" already at the string tree level. ${ }^{5}$ The volume modulus on the other hand, resides in $M_{J}$. Therefore, in type IIA $\alpha^{\prime}$ corrections appear in $M_{\mathrm{v}}$ whereas in type IIB they correct $M_{\mathrm{h}}$. This situation is identical to the situation for Calabi-Yau compactifications and is summarized in table 1 .

It is important to note that for the leading order corrections there are generically mixing terms of the form $\partial_{\mu} \phi \partial^{\mu} \ln V$ between the dilaton and the volume. For CalabiYau compactifications, the tree-level coefficent of this term has been computed in [30, 31] but the one-loop correction has only been inferred indirectly in [31] by insisting that the split (2.1) continues to hold at the loop level. To diagonalize such terms, one requires mixing between the dilaton and volume modulus in defining the combinations that appear in the appropriate vector and hypermultiplet moduli spaces. Such mixing cannot affect the leading-order contributions to the moduli space metrics but will enter at higher order.

In the following, we first consider only the metric moduli $\operatorname{Re} t^{i}$ and $z^{a}$ and use the splitting (2.1) and our assumptions about the expansion (2.10) to constrain the form of the leading corrections to the metrics on $M_{\mathrm{v}}$ and $M_{\mathrm{h}}$. We then consider the dependence of the NS $B$-field and RR moduli, using analogues of the standard Calabi-Yau compactification arguments to investigate how first the full perturbative vector-multiplet $\alpha^{\prime}$ corrections and $g_{s}$ corrections are constrained.

\subsection{Constraints on the metric moduli}

Let us first focus on the leading perturbative corrections both in $\alpha^{\prime}$ and $g_{s}$ for the metric moduli $\operatorname{Re} t^{i}$ and $z^{a}$. One can distinguish two types of terms. One is the contribution of pointlike massive string states, either in tree-level exchange or in one-loop diagrams. Given that we are expanding around a large volume limit of the compactification space, the masses of these states are much larger than the inverse radius of $X_{6}$. Hence they

\footnotetext{
${ }^{5}$ Here exact is in quotation marks since at special points in the moduli space - for example at the conifold point - non-perturbative corrections which are not governed by the dilaton can occur and correct $M_{\mathrm{v}}[41]$.
} 
are intrinsically ten-dimensional effects and correspond to a dimensional reduction of the ten-dimensional string tree-level and one-loop effective action. The second type of term is intrinsically four-dimensional and give "threshold effects" arising from integrating out heavy states coming from Kaluza-Klein modes and wound string states on $X_{6}$. For type II Calabi-Yau compactifications one notably finds that all such threshold corrections in fact vanish [28].

Before going into the details of the $\alpha^{\prime}$ and $g_{s}$ corrections of the four-dimensional theory, to be discussed in the next subsections, let us recall the structure of the higher order string tree-level and one-loop terms for the gravitational modes of the ten-dimensional effective action. They have the form (see e.g. [28, 42])

$$
\begin{aligned}
\left.\mathcal{L}_{\mathrm{IIA}}\right|_{R^{4}} & \sim \zeta(3) \mathrm{e}^{-2 \varphi}\left(t_{8} t_{8}+\frac{1}{4} \varepsilon \varepsilon\right) R^{4}+2 \zeta(2)\left(t_{8} t_{8}-\frac{1}{4} \varepsilon \varepsilon\right) R^{4}+B \wedge I_{8}, \\
\left.\mathcal{L}_{\mathrm{IIB}}\right|_{R^{4}} & \sim \zeta(3) \mathrm{e}^{-2 \varphi}\left(t_{8} t_{8}+\frac{1}{4} \varepsilon \varepsilon\right) R^{4}+2 \zeta(2)\left(t_{8} t_{8}+\frac{1}{4} \varepsilon \varepsilon\right) R^{4},
\end{aligned}
$$

where $\varphi$ is the ten-dimensional dilaton, $\zeta$ is the Riemann zeta function, and

$$
\begin{aligned}
\left(t_{8} t_{8} \pm \frac{1}{4} \varepsilon \varepsilon\right) R^{4} \equiv & \left(t^{M_{1} \cdots M_{8}} t_{N_{1} \cdots N_{8}} \pm \frac{1}{4} \varepsilon^{P Q M_{1} \cdots M_{8}} \varepsilon_{P Q N_{1} \cdots N_{8}}\right) \\
& \times R_{M_{1} M_{2}}{ }^{N_{1} N_{2}} \cdots R_{M_{7} M_{8}}{ }^{N_{7} N_{8}}
\end{aligned}
$$

$R_{M_{1} M_{2}}{ }^{N_{1} N_{2}}$ denotes the Riemann tensor, $\epsilon$ is the totally antisymmetric Levi-Civita tensor, and $t_{8}$ is antisymmetric in each successive pair of indices and symmetric under the exchange of any two pairs. Given an antisymmetric tensor $M_{M N}$, the $t$-tensor contraction reads

$$
t_{M_{1} \cdots M_{8}} M^{M_{1} M_{2}} \cdots M^{M_{7} M_{8}}=24 \operatorname{tr} M^{4}-6\left(\operatorname{tr} M^{2}\right)^{2} .
$$

We also have

$$
4608(2 \pi)^{4} I_{8}(R) \equiv t_{N_{1} \cdots N_{8}} R^{N_{1} N_{2}} \wedge \cdots \wedge R^{N_{7} N_{8}}=\left[24 \operatorname{tr} R^{4}-6\left(\operatorname{tr} R^{2}\right)^{2}\right],
$$

where $R^{N_{1} N_{2}}$ is the curvature 2 -form. Note that the supersymmetric completion of these terms will include, in the NSNS sector, higher derivative objects built from curvatures, $H=\mathrm{d} B$ and derivatives of the dilaton, which would be relevant if for instance one was interested in backgrounds with non-trivial $H$-flux. However, although recently some significant progress has been made [43], less is known about the exact form of these terms.

\subsection{1 $\alpha^{\prime}$ corrections}

Let us start by considering the leading $\alpha^{\prime}$ corrections. As usual, all the contributions arise from the ten-dimensional effective action (3.1). ${ }^{6}$ In principle, this can be derived by directly performing a Kaluza-Klein reduction of (3.1). Since $t_{8} t_{8} R^{4}$ never involves contractions of

\footnotetext{
${ }^{6}$ Note that we are expanding in terms of supersymmetry representations. Since we are off-shell these do not necessarily correspond to eigenstates of the Laplacian, and so, in contrast to the conventional case, there are potentially threshold corrections from tree-level exchange of Kaluza-Klein modes. However, by assumption the zeroth-order low-energy effective theory is a gauged supergravity for the moduli alone. This implies that the SU(3) background does not source the heavy Kaluza-Klein modes, and hence such contributions are absent. For the string winding modes, conservation of winding number means they similarly cannot be sourced. By contrast, both types of heavy modes can contribute in loops.
} 
indices on the same Riemann tensor, in the four-dimensional effective action any correction to the Einstein term must come from $\varepsilon \varepsilon R^{4}$ [28]. More precisely, the only contraction that gives a term with only two space-time derivatives is where $P Q$ in (3.2) are four-dimensional space-time indices, and the two other space-time indices on each $\varepsilon$ contract the same Riemann tensor. (If they contract different Riemann tensors one obtains higher-derivative scalar field terms.) Integrating over the internal space, we obtain

$$
\begin{aligned}
& \int_{X_{6}} \varepsilon^{\rho \sigma \mu_{1} \mu_{2}} \varepsilon^{m_{1} \cdots m_{6}} \varepsilon_{\rho \sigma \nu_{1} \nu_{2}} \varepsilon_{n_{1} \cdots n_{6}} R_{\mu_{1} \mu_{2}}{ }^{\nu_{1} \nu_{2}} R_{m_{1} m_{2}}{ }^{n_{1} n_{2}} R_{m_{3} n_{4}}{ }^{n_{3} n_{4}} R_{m_{5} n_{6}}{ }^{{ }_{5} n_{6}} \\
\sim & \mathcal{R} \int_{X_{6}}\left(\varepsilon^{m_{1} \cdots m_{6}} \varepsilon_{n_{1} \cdots n_{6}} R_{m_{1} m_{2}}{ }^{{ }_{1} n_{2}} R_{m_{3} n_{4}}{ }^{n_{3} n_{4}} R_{m_{5} n_{6}}{ }^{{ }_{5} n_{6}}\right) \sim \mathcal{R} \chi\left(X_{6}\right),
\end{aligned}
$$

where $\mathcal{R}$ is the four-dimensional Ricci scalar and $\chi\left(X_{6}\right)$ the Euler characteristic of $X_{6}$. Thus we see that for $\mathrm{SU}(3)$ structures, the correction to the Ricci scalar term comes only from $\varepsilon \varepsilon R^{4}$ and is proportional to $\chi$ exactly as for Calabi-Yau compactifications. ${ }^{7}$

For the scalar kinetic energy corrections, we note that $\varepsilon \varepsilon R^{4}$ necessarily has four spacetime indices and thus cannot contribute. One can, however, get a correction from the $t_{8} t_{8} R^{4}$ terms $[28,30,44]$. We will denote these corrections to the metrics on the $M_{J}$ and $M_{\Omega}$ moduli spaces as $\delta g_{J}^{\text {tree }}$ and $\delta g_{\Omega}^{\text {tree }}$, respectively, and the leading order, uncorrected metrics as $g_{J}^{0}$ and $g_{\Omega}^{0}$. Inspecting the terms in (3.1), we can now write the leading $\alpha^{\prime}$ correction to both the IIA and IIB Lagrangians in the string frame as [31]

$$
\begin{aligned}
& \Delta \mathcal{L}_{\text {tree }} \sim \mathrm{e}^{-2 \phi}\left(1+V^{-1} c\right) \mathcal{R} \\
& \\
&+\mathrm{e}^{-2 \phi}\left(\operatorname{Re} g_{J}^{0}+V^{-1} \operatorname{Re} \delta g_{J}^{\text {tree }}\right)_{i j} \partial_{\mu} v^{i} \partial^{\mu} v^{j} \\
&+\mathrm{e}^{-2 \phi}\left(g_{\Omega}^{0}+V^{-1} \delta g_{\Omega}^{\text {tree }}\right)_{a \bar{b}} \partial_{\mu} z^{a} \partial^{\mu} \bar{z}^{\bar{b}}+\ldots,
\end{aligned}
$$

where $v^{i}=\operatorname{Re} t^{i}, \phi$ is the four-dimensional dilaton defined as $\mathrm{e}^{2 \phi}=V^{-1} \mathrm{e}^{2 \varphi}$ and

$$
c=\frac{2 \zeta(3)}{(2 \pi)^{3}} \chi\left(X_{6}\right) .
$$

The next step is to Weyl-rescale to the Einstein frame and then expand in terms of $\mathrm{e}^{2 \phi}$ and the inverse volume $V^{-1}$, which parametrize loop and $\alpha^{\prime}$ corrections, respectively. ${ }^{8}$ To leading order in $V^{-1}$, one finds

$$
\begin{aligned}
\Delta \mathcal{L}_{\text {tree }} \sim \mathcal{R} & +\left(\operatorname{Re} g_{J}^{0}+V^{-1}\left(\delta g_{J}^{\text {tree }}-c \operatorname{Re} g_{J}^{0}\right)\right)_{i j} \partial_{\mu} v^{i} \partial^{\mu} v^{j} \\
& +\left(g_{\Omega}^{0}+V^{-1}\left(\delta g_{\Omega}^{\text {tree }}-c g_{\Omega}^{0}\right)\right)_{a \bar{b}} \partial_{\mu} z^{a} \partial^{\mu} \bar{z}^{\bar{b}}+\ldots
\end{aligned}
$$

Recalling that there can be no $\alpha^{\prime}$ corrections to the complex structure moduli space we immediately have

$$
\delta g_{\Omega}^{\text {tree }}=c g_{\Omega}^{0} .
$$

\footnotetext{
${ }^{7}$ The fact that this property does not only hold for Calabi-Yau compactifications has also been observed in $[23]$.

${ }^{8}$ Just to reiterate, the definitions of both the four-dimensional dilaton $\phi$ and the volume $V$ change due to their mixing just discussed [30,31]. However this, and any more general mixing that might appear for generic $\mathrm{SU}(3)$ backgrounds, does not change the corrections to the moduli space metrics at this order.
} 
One might be tempted to argue, for example by invoking a putative mirror symmetry, that $\operatorname{Re} \delta g_{J}$ is similarly proportional to $\operatorname{Re} g_{J}^{0}$, as it is in the Calabi-Yau case. In the next section, by considering the contributions of the imaginary parts of the moduli coming from the NS $B$-field, we will see that this is indeed the case.

\subsection{2 $g_{s}$ corrections}

For loop corrections both ten-dimensional and threshold contributions are relevant, and a priori - there is little one can deduce about the form of the four-dimensional low-energy action. Before Weyl-rescaling the loop-corrected Lagrangians have the form

$$
\begin{aligned}
\Delta \mathcal{L}_{\text {loop }}^{\text {IIA } / \text { IIB }} \sim & \left(V \mathrm{e}^{-2 \varphi}+f^{\mathrm{A} / \mathrm{B}}\right) \mathcal{R}+\left(V \mathrm{e}^{-2 \varphi} \operatorname{Re} g_{J}^{0}+\operatorname{Re} \delta g_{J}^{\text {loop }, \mathrm{A} / \mathrm{B}}\right)_{i j} \partial_{\mu} v^{i} \partial^{\mu} v^{j} \\
& +\left(V \mathrm{e}^{-2 \varphi} g_{\Omega}^{0}+\delta g_{\Omega}^{\text {loop }, \mathrm{A} / \mathrm{B}}\right)_{a \bar{b}} \partial_{\mu} z^{a} \partial^{\mu} \bar{z}^{\bar{b}}+\ldots,
\end{aligned}
$$

where $\delta g_{J}^{\text {loop,A/B }}$ and $\delta g_{\Omega}^{\text {loop,A/B }}$ denote the leading order loop corrections of the two metrics while $f^{\mathrm{A} / \mathrm{B}}$ parametrize the loop-correction to the Einstein term. All three corrections contain a contribution from the reduction of the ten-dimensional terms given in (3.1) and in principle an additional threshold correction. After rescaling to the Einstein frame one finds

$$
\begin{aligned}
\Delta \mathcal{L}_{\text {loop }}^{\text {IIA } / \mathrm{IIB}} \sim \mathcal{R} & +\left(\operatorname{Re} g_{J}^{0}+\mathrm{e}^{2 \phi}\left(\operatorname{Re} \delta g_{J}^{\text {loop }, \mathrm{A} / \mathrm{B}}-f^{\mathrm{A} / \mathrm{B}} \operatorname{Re} g_{J}^{0}\right)\right)_{i j} \partial_{\mu} v^{i} \partial^{\mu} v^{j} \\
& +\left(g_{\Omega}^{0}+\mathrm{e}^{2 \phi}\left(\delta g_{\Omega}^{\text {loop }, \mathrm{A} / \mathrm{B}}-f^{\mathrm{A} / \mathrm{B}} g_{\Omega}^{0}\right)\right)_{a \bar{b}} \partial_{\mu} z^{a} \partial^{\mu} \bar{z}^{\bar{b}}+\ldots
\end{aligned}
$$

The requirement that there be no loop corrections to the vector multiplet moduli means that

$$
\operatorname{Re} \delta g_{J}^{\text {loop, } \mathrm{A}}=f^{\mathrm{A}} \operatorname{Re} g_{J}^{0}, \quad \delta g_{\Omega}^{\text {loop, } \mathrm{B}}=f^{\mathrm{B}} g_{\Omega}^{0} .
$$

Since $g_{J}$ can only depend on the $v^{i}$ and $g_{\Omega}$ can only depend on the $z^{a}$ we can infer that $f^{\mathrm{A}}$ is a function of only $v^{i}$ and $f^{\mathrm{B}}$ is a function of only $z^{a}$.

At this point we can put no further constraints on the corrections. However, it is interesting to note that for Calabi-Yau compactifications the type IIA and IIB corrections are related by

$$
f^{\mathrm{A}}=-f^{\mathrm{B}}, \quad \operatorname{Re} \delta g_{J}^{\text {loop,A }}=\operatorname{Re} \delta g_{J}^{\text {loop, } \mathrm{B}}, \quad \delta g_{\Omega}^{\text {loop, } \mathrm{A}}=\delta g_{\Omega}^{\text {loop,B }} .
$$

These relations arise by noting from which worldsheet spin-structure sectors the terms originate [28]. The Einstein term corrections come only from the odd-odd sector, while the metric corrections come from the even-even sector. Since $t_{8}$ is even under parity, one sees that in the ten-dimensional action (3.1), the one-loop $\epsilon \epsilon$ term is odd-odd, and changes sign between IIA and IIB, while the $t_{8} t_{8}$ term is even-even and is the same for both IIA and IIB.

Using (3.5), we see that the loop corrections which arise from solely reducing the terms in the ten-dimensional action given in (3.1) do respect the relations (3.13) even for SU(3)structure backgrounds. Therefore, with the additional assumption that the same is true for the threshold contributions, one obtains

$$
\operatorname{Re} \delta g_{J}^{\text {loop, } \mathrm{A}}=\operatorname{Re} \delta g_{J}^{\text {loop, } \mathrm{B}}=f \operatorname{Re} g_{J}^{0}, \quad \delta g_{\Omega}^{\text {loop,A }}=\delta g_{\Omega}^{\text {loop, } \mathrm{B}}=-f g_{\Omega}^{0},
$$


where $f=f^{\mathrm{A}}=-f^{\mathrm{B}}$. Furthermore, since the corrections to Re $g_{J}$ can only depend on the moduli Re $t^{i}$ and the corrections to $g_{\Omega}$ can only depend on the moduli $z^{a}$, we also have that

$$
f=\text { constant }
$$

Note that we would have come to the same conclusions by assuming that there is a mirror symmetry between IIA and IIB sending $f$ to $-f$. In fact, as we will see in section 3.3 , we are also led to something very close to the relations (3.14) once we consider the form of the RR kinetic terms in the hypermultiplet sector, without needing the assumption about threshold corrections or mirror symmetry made here.

\subsection{Perturbative $\alpha^{\prime}$ corrections}

As discussed in section 3.1.1, while there are necessarily no $\alpha^{\prime}$ corrections to $g_{\Omega}$, we could say nothing concrete about the corrections to $g_{J}$. However, we did not consider higherderivative couplings of the NS $B$-field as they are not yet completely known [43]. Let us now address that issue and see how it might allow us to also constrain the perturbative corrections to $g_{J}$.

In the reduction, the light modes of $B$ combine with the deformation of $J$, as in (2.10), to form complex scalar coordinates $t^{i}$ on $M_{J}$. The $\mathcal{N}=2$ prepotential $\mathcal{F}$ that determines the special-Kähler metric depends holomorphically on $t^{i}$ and is given in the large volume limit — so as to match (2.8) - by

$$
\mathcal{F}_{0}(t)=\kappa_{i j k} t^{i} t^{j} t^{k}
$$

with $\kappa_{i j k}$ real constants and we take $X^{0}=\mathrm{i}$ and $X^{i}=t^{i}$. (In Calabi-Yau compactifications the $\kappa_{i j k}$ are the classical intersection numbers; more generally they are related to the basis forms $\omega_{i}[14,15]$.) In order to determine or constrain perturbative $\alpha^{\prime}$ corrections, we have to determine the sub-leading corrections to $\mathcal{F}$. Expanding in large $t^{i}$ we have generically

$$
\mathcal{F}(t)=\mathcal{F}_{0}(t)+\alpha_{i j} t^{i} t^{j}+\beta_{i} t^{i}+\gamma+\hat{\mathcal{F}}(t)
$$

where $\hat{\mathcal{F}}$ contains non-perturbative corrections (i.e. instanton corrections) together with possibly negative powers of $t^{i}$. Here we use the fact that each power in the $\alpha^{\prime}$ expansion comes with a volume factor $V^{-1 / 3}$. From (2.8) we see that $V$ is cubic in $t^{i}$

$$
V=\frac{1}{6} \kappa_{i j k}(t+\bar{t})^{i}(t+\bar{t})^{j}(t+\bar{t})^{k}=\frac{1}{6} \mathrm{e}^{-K_{0}}
$$

where $K_{0}$ is the leading order Kähler potential computed from $\mathcal{F}_{0}$, and hence the corrections to $\mathcal{F}_{0}$ are in descending powers of $t^{i}$.

Inserting (3.17) into (2.3) one obtains

$$
\mathrm{e}^{-K}=\kappa_{i j k}(t+\bar{t})^{i}(t+\bar{t})^{j}(t+\bar{t})^{k}+a_{i j} t^{i} \bar{t}^{j}+\mathrm{i} b_{i}(t-\bar{t})^{i}+c+\ldots,
$$

where

$$
a_{i j}=2 \mathrm{i}\left(\alpha_{i j}-\bar{\alpha}_{i j}\right), \quad \mathrm{i} b_{i}=\left(\beta_{i}-\bar{\beta}_{i}\right), \quad c=2 \mathrm{i}(\gamma-\bar{\gamma}) .
$$


So we see that the real parts of $\alpha_{i j}, \beta_{i}$ and $\gamma$ actually do not enter the Kähler potential and for our purpose may be set to zero without loss of generality. ${ }^{9}$ Computing the metric from (3.19) we find

$$
g_{J i \bar{j}}=-\mathrm{e}^{K}\left(6 \kappa_{i j}+a_{i j}\right)+\mathrm{e}^{2 K}\left(3 \kappa_{i}+a_{i k} \bar{t}^{k}+\mathrm{i} b_{i}\right)\left(3 \kappa_{j}+a_{j k} t^{k}-\mathrm{i} b_{j}\right),
$$

where we abbreviated

$$
\kappa_{i j}=\kappa_{i j k}(t+\bar{t})^{k}, \quad \kappa_{i}=\kappa_{i l k}(t+\bar{t})^{l}(t+\bar{t})^{k} .
$$

We would like to determine the values for $a_{i j}, b_{i}$ and $c$. Recall that for Calabi-Yau compactifications there is a perturbative Peccei-Quinn symmetry $B \rightarrow B+\lambda^{i} \omega_{i}$, which implies that $g_{i \bar{j}}^{J}$ has an isometry $t^{i} \rightarrow t^{i}+\mathrm{i} \lambda^{i}$ for constant real $\lambda^{i}$. This is very constraining, implying in particular that $a_{i j}=b_{i}=0$. In fact, the PQ symmetry together with the large volume limit is enough to also prove that no negative powers of $t^{i}$ can appear in $\mathcal{F}$ and that the perturbative prepotential can only be $\mathcal{F}(t)=\mathcal{F}_{0}(t)+\gamma$ with $\gamma$ constant. ${ }^{10}$

Now let us turn to the general SU(3) structure case. In general the PQ symmetry is a priori not present since the basis forms are not necessarily closed. However, as discussed in the previous sub-section, the leading string corrections to supergravity, including those involving $B$, appear at order $\alpha^{\prime 3}$. Thus all leading corrections to $g_{i \bar{j}}^{J}$ are suppressed by a factor of $V^{-1}$ and hence by cubic powers in $t^{i}$. This means, simply by counting powers of $t$, that we expect $a_{i j}=b_{i}=0$ and in fact, somewhat surprisingly, the leading order corrected metric still has the PQ symmetry.

In the analysis so far we made an implicit assumption since the expansion (3.17) in $V^{-1 / 3}$ strictly defines $\mathcal{F}$ as a sum of functions homogeneous under the rescaling $t^{i} \rightarrow \mu t^{i}$, that is

$$
\mathcal{F}(t)=\mathcal{F}_{0}(t)+\mathcal{F}_{1}(t)+\mathcal{F}_{2}(t)+\cdots+\mathcal{F}_{n p}(t),
$$

where $\mathcal{F}_{i}(t)$ scales as $\mu^{3-i}$ and $\mathcal{F}_{n p}(t)$ is the non-perturbative correction. Thus in (3.17) we are really extracting only the polynomial parts of each $\mathcal{F}_{i}(t)$ in the expansion, relegating the non-polynomial contributions to $\hat{\mathcal{F}}$. The latter would signal power-like singularities in the variables $t^{i}$ and naively one might expect their absence also in the $\mathrm{SU}(3)$ structure case, although notably, under T-duality, the contributions of wound strings are precisely of this type. For the leading $\alpha^{\prime}$ corrections we can actually address this question directly. First, as for the $\alpha$ and $\beta$ terms above, since the leading string corrections to supergravity, including those involving the $B$-field, appear at order $\alpha^{\prime 3}$, we can immediately argue that $\mathcal{F}_{1}$ and $\mathcal{F}_{2}$ vanish, since they correspond to $\alpha^{\prime}$ and $\alpha^{\prime 2}$ corrections. Turning to the $\alpha^{\prime 3}$ correction, $\mathcal{F}_{3}$ we have already noted that the corrections to the kinetic terms are unaffected by moduli mixing to this order. Thus we can take the zeroth-order definitions, and identify $\operatorname{Im} t^{i}$

\footnotetext{
${ }^{9}$ In Calabi-Yau compactifications they are determined to be non-vanishing using mirror symmetry, but this plays no role in the following.

${ }^{10}$ To see this one inserts an arbitrary function into (2.3), computes the metric and imposes the PQ symmetry - this determines $\mathcal{F}(t)=\mathcal{F}_{0}(t)+\gamma$ (modulo terms that do not contribute to $K$ ). In particular, the term $\hat{\mathcal{F}}$ in (3.17) cannot have negative powers of $t^{i}$ and only contains non-perturbative corrections which break the PQ symmetry to a discrete subgroup allowing for the dependence $\hat{\mathcal{F}}\left(e^{2 \pi t}\right)$.
} 
with the expansion of the $B$-field in (2.10). Next we note, just by power counting, that the leading higher-derivative corrections to the ten-dimensional effective action can include only finite powers of $H=\mathrm{d} B$ up to $H^{4}$. Hence we can only have polynomial dependence on $\operatorname{Im} t^{i}$ in the correction to the metric $\delta g_{J}$. Together with the homogeneity condition this is enough to argue that in fact $\mathcal{F}_{3}$ is constant.

To summarize, for any $\mathrm{SU}(3)$ structure compactifications we have argued that the $\alpha^{\prime}$-corrected prepotential on $M_{J}$ has the form

$$
\mathcal{F}=\kappa_{i j k} t^{i} t^{j} t^{k}-\frac{1}{2} \mathrm{i} \tilde{c}
$$

where $\tilde{c}$ is constant. Note that this is exactly the same form as for Calabi-Yau compactifications, in which case $\tilde{c}=2 \zeta(3) \chi\left(X_{6}\right) /(2 \pi)^{3}$ is proportional to the Euler characteristic (and equal to $c$ in (3.7)). The corresponding metric is given by

$$
g_{J i \bar{j}}=-6 \mathrm{e}^{K} \kappa_{i j}+9 \mathrm{e}^{2 K} \kappa_{i} \kappa_{j}, \quad \text { with } \quad \mathrm{e}^{-K}=\kappa_{i j k}(t+\bar{t})^{i}(t+\bar{t})^{j}(t+\bar{t})^{k}+\tilde{c},
$$

so that expanding to first order in $V^{-1}$ gives

$$
g_{J i \bar{j}}=g_{J i \bar{j}}^{0}+\mathrm{e}^{K_{0}} \delta g_{J i \bar{j}}
$$

where $g_{J i \bar{j}}^{0}$ and $K_{0}$ are the leading order metric and Kähler potential computed from the cubic prepotential $\mathcal{F}_{0}$ given in $(3.16)$ and ${ }^{11}$

$$
\delta g_{J i \bar{j}}=-\tilde{c} g_{J i \bar{j}}^{0}-9 \tilde{c} \mathrm{e}^{2 K_{0}} \kappa_{i} \kappa_{j}
$$

This result might seem somewhat surprising as we just argued that the PQ isometries are not present in $\mathrm{SU}(3)$ structure compactifications, yet the metric given in (3.25) does have them. This is arising because the ten-dimensional corrections have a very particular universal form, which together with $\mathcal{N}=2$ supersymmetry imply the absence of subleading polynomial corrections in the prepotential. In that sense the PQ isometries can be viewed as "accidental" symmetries. (Of course they are broken in the potential already at leading order [14].)

The arguments used so far did not determine the value of $\tilde{c}$ for $\mathrm{SU}(3)$ structure compactifications. It is tempting to conjecture that it is again equal to $c$ in (3.7) and hence given by the Euler characteristic. This would be consistent with any putative mirror symmetry given the relation (3.9). It is also interesting to note that it is consistent with the arguments of [45], where it was shown that manifolds $X_{6}$ with vanishing Euler characteristic necessarily have an additional SU(2) structure and the compactification can be viewed as a spontaneously broken $N=4$ theory. The $N=4$ supersymmetry then forbids any

\footnotetext{
${ }^{11}$ Note that the second term appears to violate the conjecture in section 3.1 that the contributions to $\delta g_{J}^{\text {tree }}$ and $\delta g_{\Omega}^{\text {tree }}$ from the $t_{8} t_{8} R^{4}$ term are proportional to $g_{J}^{0}$ and $g_{\Omega}^{0}$ respectively. However just as in the Calabi-Yau case [30, 31], the conjecture is really that these are the forms of the contributions up to pieces arising from a total derivative term in the expansion of $t_{8} t_{8} R^{4}$. The point is that integrating such a term by parts against the $V^{-1} \mathrm{e}^{-2 \phi}$ coefficient in (3.6) generates the second term in (3.27) for $\delta g_{J}$ but no such term for $\delta g_{\Omega}$. The corresponding term is also absent for $\delta g_{J}^{\text {loop }}$ in the reduction of the ten-dimensional loop correction, since there the coefficient is independent of $V$.
} 
perturbative corrections and implies $\tilde{c} \sim \chi\left(X_{6}\right)$ or in other words that $\tilde{c}$ must vanish as the Euler characteristic goes to zero. ${ }^{12}$

One might also wonder if, as in the Calabi-Yau case, this leading contribution actually gives the perturbative $\alpha^{\prime}$ correction to all orders. If the argument that $\mathcal{F}_{3}$ is polynomial in $\operatorname{Im} t^{i}$ can be extended to all $\mathcal{F}_{n}$ then homogeneity implies that all the higher $\mathcal{F}_{n}$ with $n>3$ do indeed vanish. However, although one can again argue that the corresponding higher-derivative corrections must be polynomial in $H$, the fact that there may be moduli mixing means that we cannot conclude that $\mathcal{F}_{n}$ is generally polynomial in $\operatorname{Im} t^{i}$. Thus, as stands, we cannot argue against higher-order corrections.

\subsection{Perturbative $g_{s}$ corrections}

As we discussed above, for Calabi-Yau compactifications the zero-modes $\xi, \tilde{\xi}$ of the RR potentials $C_{p}$ together with the axion $\sigma$ in the hypermultiplet moduli space admit $2 n_{\mathrm{h}}+1$ (perturbative) Peccei-Quinn shift symmetries given in (2.14). In SU(3) compactifications the situation is more involved since generically some of the scalar fields become massive, or in other words the number of the zero modes is reduced, because the basis forms are no longer harmonic. As discussed in $[4,5,14,15,18]$, a subset of the shift symmetries (2.14) turns local in that $c^{I}, \tilde{c}_{I}$ become space-time dependent and appropriate couplings to the gauge fields are induced. $\mathcal{N}=2$ supersymmetry in turn demands a non-trivial scalar potential which lifts some of the flat directions corresponding to the "non-zero" but light modes mentioned above. An argument along the lines of ref. [46] further shows that these gauged isometries survive after including perturbative and non-perturbative corrections.

The number of gauged isometries depends on the specific structure of the non-trivial torsion, that is, the constants $e_{i I}$ and $p_{i}^{I}$ in (2.11). Nevertheless, one can determine that for any $\mathrm{SU}(3)$ compactification at least $n_{\mathrm{h}}$ of the isometries in (2.14) survive perturbatively, and only the question which are gauged depends on the details of $X_{6}$ [15]. To review this argument, note that in this case, for the RR scalars in (2.13),

$$
\begin{aligned}
\mathrm{d}\left(\xi^{I} \alpha_{I}+\tilde{\xi}_{I} \beta^{I}\right) & =\left(\mathrm{d} \xi^{I}\right) \alpha_{I}+\left(\mathrm{d} \tilde{\xi}_{I}\right) \beta^{I}+\left(\xi^{I} e_{i I}+\tilde{\xi}_{I} p_{i}^{I}\right) \tilde{\omega}^{i} \\
\mathrm{~d}\left(\xi^{0}+\xi^{i} \omega_{i}+\tilde{\xi}_{i} \tilde{\omega}^{i}+\tilde{\xi}_{0} \varepsilon\right) & =\mathrm{d} \xi^{0}+\left(\mathrm{d} \xi^{i}\right) \omega_{i}+\left(\mathrm{d} \tilde{\xi}_{i}\right) \tilde{\omega}^{i}+\left(\mathrm{d} \tilde{\xi}_{0}\right) \varepsilon+\left(\xi^{i} e_{i I}\right) \alpha^{I}-\left(\xi^{i} p_{i}^{I}\right) \beta_{I} .
\end{aligned}
$$

This means that the RR field strengths depend explicitly on the combinations $\xi^{I} e_{i I}+\tilde{\xi}_{I} p_{i}^{I}$ in type IIA and $\xi^{i} e_{i I}$ and $\xi^{i} p_{i}^{I}$ in type IIB. Thus for IIB it is clear that at most one loses the $n_{h}-1$ isometries $\xi^{i} \rightarrow \xi^{i}+c^{i}$. For type IIA, one notes that $\mathrm{d}^{2}=0$ implies $e_{i I} p_{j}^{I}-p_{i}^{I} e_{j I}=0$ and hence the vectors $Z_{i}=\left(e_{i I}, p_{i}^{I}\right)$ span an isotropic subspace of the $2 n_{h}$-dimensional symplectic space spanned by $\alpha_{I}$ and $\beta^{I}$. Thus there can be at most $n_{h}$ linearly independent $Z_{i}$ and hence at most $n_{h}$ combinations $\xi^{I} e_{i I}+\tilde{\xi}_{I} p_{i}^{I}$ that appear explicitly in the RR field strengths and hence have broken PQ symmetry. This result implies that all hypermultiplets can be dualized to tensor multiplets, where the scalars which transform as in (2.14) are replaced by dual antisymmetric tensors [47-49]. ${ }^{13}$

\footnotetext{
${ }^{12}$ We thank H. Triendl for pointing this out.

${ }^{13}$ In type IIB the tensor multiplets already arise in the field basis which naturally occurs in the KaluzaKlein reduction [50].
} 
This property was used in [32] to parameterize the possible perturbative corrections in Calabi-Yau compactifications in terms of one scalar function $\Delta(z) . \mathcal{N}=2$ supersymmetry alone already constrains the string loop-corrected scalar field spaces to be quaternionicKähler. Our assumption of SU(3) structure further implies that $M_{\mathrm{h}}$ is a torus fibration over a special-Kähler manifold. However, because not all the RR isometries survive, the metric on $M_{\mathrm{h}}$ no longer has to be 'special quaternionic-Kähler', or in other words the loop-corrected metric is generically not of the Ferrara-Sabharwal form given in (2.5) with merely a loop-corrected prepotential $\mathcal{G}$. However, the existence of at least $n_{\mathrm{h}}+1$ unbroken translational isometries additionally constrains the form of the metric [27, 29, 32], and this is best described in terms of the dual tensor multiplets. For Calabi-Yau compactifications - and as we just argued, also for $\mathrm{SU}(3)$ structure compactifications - all hypermultiplets can be dualized to tensor multiplets, and as a consequence the constraints determined in $[47-49,51]$ apply. Using the additional property that the dilaton organizes the $g_{s}$ expansion, we can repeat the analysis performed in [32] and arrive at the same result that the corrections to $M_{\mathrm{h}}$ are determined by a single function $\Delta$, that is the imaginary part of a holomorphic function of the base-coordinates $z$ and is homogeneous of degree zero. Explicitly, the correction to (2.5) has to be of the form [32]

$$
\begin{aligned}
\mathcal{L}= & \frac{1+2 \Delta \mathrm{e}^{2 \phi}}{1+\Delta \mathrm{e}^{2 \phi}}\left(\partial_{\mu} \phi\right)^{2}+\frac{1+\Delta \mathrm{e}^{2 \phi}}{1+2 \Delta \mathrm{e}^{2 \phi}} \mathrm{e}^{4 \phi}\left(D_{\mu} \sigma-\xi^{I} D_{\mu} \tilde{\xi}_{I}-\Delta \mathcal{A}_{\mu}\right)^{2} \\
& +\left(1+\Delta \mathrm{e}^{2 \phi}\right) g_{a \bar{b}} \partial_{\mu} z^{a} \partial^{\mu} \bar{z}^{\bar{b}}-\frac{\mathrm{e}^{4 \phi}}{1+\Delta \mathrm{e}^{2 \phi}} \tilde{\mathcal{A}}_{\mu} \tilde{\mathcal{A}}^{\mu}+\Delta Y_{I J} D_{\mu} \xi^{I} D^{\mu} \xi^{J} \\
& -\mathrm{e}^{2 \phi} \mathcal{T}^{I J}\left(D_{\mu} \tilde{\xi}_{I}-2 \mathrm{i} \mathcal{M}_{I K} D_{\mu} \xi^{K}\right)\left(D^{\mu} \tilde{\xi}_{J}+2 \mathrm{i} \overline{\mathcal{M}}_{J L} D^{\mu} \xi^{L}\right)
\end{aligned}
$$

where we included appropriate covariant derivatives. $\mathcal{A}_{\mu}=-\operatorname{Im}\left(\partial_{a} \mathcal{K} \partial_{\mu} z^{a}\right)$ is the Kähler connection on the special-Kähler base, $\tilde{\mathcal{A}}_{\mu}=\operatorname{Re}\left(\partial_{a} \Delta \partial_{\mu} z^{a}\right)$, and $\mathcal{M}_{I J}(z, \bar{z}, \phi), \mathcal{T}_{I J}(z, \bar{z}, \phi)$ are quantum deformations of $\mathcal{N}_{I J}$ (defined in (2.6)) and $2 \operatorname{Re} \mathcal{N}_{I J}$, respectively. All these corrections are proportional to powers of $\Delta$ and the precise expressions of $\mathcal{M}_{I J}(z, \bar{z}, \phi)$, $\mathcal{T}_{I J}(z, \bar{z}, \phi)$ and the matrix $Y_{I J}(z, \bar{z}, \phi)$ can be found in [32]. Here we have written $z^{a}$ for the coordinates on the base, corresponding to type IIA. In type IIB these would be replaced by $t^{i}$.

Again, we can compare this form of the metric directly with the loop corrections to $M_{J}$ and $M_{\Omega}$ we discussed in section 3.1.2. It provides strong constraints on their form. Comparing (3.11) with the leading-order kinetic terms for $z^{a}$ in IIA and for $t^{i}$ in IIB in $(3.29)$, we see that

$$
\delta g_{\Omega}^{\text {loop }, \mathrm{A}}=\left(\Delta^{\mathrm{A}}+f^{\mathrm{A}}\right) g_{\Omega}^{0}, \quad \delta g_{J}^{\text {loop }, \mathrm{B}}=\left(\Delta^{\mathrm{B}}+f^{\mathrm{B}}\right) g_{J}^{0},
$$

where $\Delta^{\mathrm{A}}$ and $\Delta^{\mathrm{B}}$ are the relevant functions of $z^{a}$ and $t^{i}$ respectively. Again, we see that the corrections are in fact proportional to the zeroth-order metrics, consistent with the conjecture (3.14). Furthermore, given the relations (3.12) and the fact that the corrections to $g_{\Omega}$ and $g_{J}$ can only depend on $z^{a}$ and $t^{i}$ respectively, we can also conclude that

$$
f^{\mathrm{A}}=\text { constant }, \quad f^{\mathrm{B}}=\text { constant } .
$$


This implies that in both type IIA and IIB the renormalization of the Einstein term (including the threshold corrections) is simply given by a constant.

Also in the hypermultiplet sector we see accidental PQ symmetries. Although the expansion is not generically compatible with preserving all $2 n_{\mathrm{h}}+1 \mathrm{PQ}$ symmetries, this is not realized in the correction to the hypermultiplet moduli space - the corrected metric (3.29) still preserves all the shift symmetries. Instead, the breaking is only realized in the mass terms. Furthermore, it was argued in [32] that the tensor multiplet structure and the dilaton expansion was enough to exclude any further corrections beyond one-loop. Using the same logic here, the implication is that for $\mathrm{SU}(3)$ structure compactifications there is a non-renormalization theorem stating that the hypermultiplet metric can only be corrected perturbatively at one-loop but not beyond.

As stands we are still left with arbitrary functions $\Delta^{\mathrm{A}}(z)$ and $\Delta^{\mathrm{B}}(t)$. However, as discussed in section 3.1, a putative mirror symmetry points to the conjecture that the correction terms respect the same spin-structure symmetries as in Calabi-Yau compactifications and both $\Delta^{\mathrm{A}}$ and $\Delta^{\mathrm{B}}$ are in fact constant. If in addition we invoke the arguments of [45] we can further constrain the constant to be proportional to the Euler characteristic $\chi\left(X_{6}\right)$. We therefore close with the conjecture

$$
\Delta^{\mathrm{A}}=-\Delta^{\mathrm{B}}=\text { constant } \sim \chi\left(X_{6}\right) .
$$

\section{Summary and outlook}

We have constrained the leading perturbative $\alpha^{\prime}$ and $g_{s}$ corrections to the moduli space metric arising in compactifications of type II theories on six-dimensional SU(3) structure manifolds $X_{6}$, subject to some simple assumptions about the low-energy modes. We have shown that both the $\alpha^{\prime}$ and $g_{s}$ leading corrections to the four-dimensional curvature scalar are constant, with the tree level contribution given by the Euler characteristic $\chi\left(X_{6}\right)$. Furthermore, the leading tree-level correction of the Kähler moduli space metric corresponds to a constant term $\tilde{c}$ in the prepotential $\mathcal{F}$ (cf. (3.24)). For the loop corrections we argued that a non-renormalization theorem holds in that the metric in the hypermultiplet sector is corrected at one-loop but receives no further perturbative correction. For the one-loop corrections we could not show that they coincide for type IIA and type IIB but they might differ by a moduli dependent function $\Delta^{\mathrm{A} / \mathrm{B}}$ (cf. (3.12), (3.30)). We summarize these results in table $2 .{ }^{14}$

While our analysis leaves $\tilde{c}$ and $\Delta^{\mathrm{A} / \mathrm{B}}$ undetermined, we were able to gather a variety of arguments which point to the fact that they are exactly as in Calabi-Yau compactifications, namely that $\Delta^{\mathrm{A} / \mathrm{B}}$ is constant and all constants are proportional to the Euler characteristic

$$
\tilde{c} \sim \Delta^{\mathrm{A} / \mathrm{B}} \sim \chi\left(X_{6}\right) .
$$

This would also imply that, even though the mass terms originating from the gaugings break some of the PQ shift symmetries, the perturbatively corrected kinetic terms actually

\footnotetext{
${ }^{14}$ Note that here we are presenting these corrections after Weyl-rescaling to the Einstein frame, in constrast to the expressions in section 3.1. Also, the full tree-level correction to $\delta g_{J}$ is given by (3.27). For simplicity, we use a shorthand of only writing the first term in (3.27) (see comment in footnote 11).
} 


\begin{tabular}{|c|c|c|c|c|}
\hline \multirow{2}{*}{} & \multicolumn{2}{|c|}{$\delta g_{J}$} & \multicolumn{2}{c|}{$\delta g_{\Omega}$} \\
\cline { 2 - 5 } & tree & loop & tree & loop \\
\hline IIA & $-\tilde{c} g_{J}^{0}$ & 0 & 0 & $\Delta^{\mathrm{A}} g_{\Omega}^{0}$ \\
\hline IIB & $-\tilde{c} g_{J}^{0}$ & $\Delta^{\mathrm{B}} g_{J}^{0}$ & 0 & 0 \\
\hline
\end{tabular}

Table 2. Summary of metric corrections.

possess all shift symmetries. In fact, the derivation of (3.29) in [32] shows that this property holds even for non-constant functions $\Delta^{\mathrm{A} / \mathrm{B}}$ under some assumptions on their pole structure.

A key open question is how dependent these results are on our assumptions about the low-energy modes, including the absence of light spin-3/2 particles. We hope nonetheless that this work provides a useful basis point for studying the form of general corrections to non-Calabi-Yau compactifications. We also have only considered the case of $\mathrm{SU}(3)$ structure manifolds, without background fluxes. An obvious extension of our results would be to consider more general $\mathrm{SU}(3) \times \mathrm{SU}(3)$-structure manifolds, and/or NSNS and RR fluxes. One could also try to investigate the form of the non-perturbative corrections. We hope to make progress on these issues in the near future.

\section{Acknowledgments}

We have benefited from conversations and correspondence with R. Minasian, B. Pioline, F. Saueressig, H. Triendl, S. Vandoren, P. Vanhove and O Varela. We also thank the referee of our paper for several helpful comments and for raising some interesting further points. J.L. thanks the Weizmann Institute and the Theory Group at CERN for their kind hospitality during the final stages of this work. This work was supported in part by the joint Network Grant DFG/LU/419/9-1 and EPSRC EP/I02784X/1, the ERC Starting Grant 259133 - ObservableString, the German-Israeli Foundation for Scientific Research and Development (GIF I-1-03847.7/2009), the I-CORE program of the Planning and Budgeting Committee and the Israel Science Foundation (grant number 1937/12), the EPSRC Programme Grant "New Geometric Structures from String Theory" EP/K034456/1 and the STFC Consolidated Grant ST/J0003533/1.

Open Access. This article is distributed under the terms of the Creative Commons Attribution License (CC-BY 4.0), which permits any use, distribution and reproduction in any medium, provided the original author(s) and source are credited.

\section{References}

[1] J.P. Gauntlett, N. Kim, D. Martelli and D. Waldram, Five-branes wrapped on SLAG three cycles and related geometry, JHEP 11 (2001) 018 [hep-th/0110034] [INSPIRE].

[2] J.P. Gauntlett, D. Martelli, S. Pakis and D. Waldram, G structures and wrapped NS5-branes, Commun. Math. Phys. 247 (2004) 421 [hep-th/0205050] [INSPIRE]. 
[3] J.P. Gauntlett, D. Martelli and D. Waldram, Superstrings with intrinsic torsion, Phys. Rev. D 69 (2004) 086002 [hep-th/0302158] [INSPIRE].

[4] S. Gurrieri, J. Louis, A. Micu and D. Waldram, Mirror symmetry in generalized Calabi-Yau compactifications, Nucl. Phys. B 654 (2003) 61 [hep-th/0211102] [INSPIRE].

[5] S. Gurrieri and A. Micu, Type IIB theory on half flat manifolds, Class. Quant. Grav. 20 (2003) 2181 [hep-th/0212278] [INSPIRE].

[6] N.J. Hitchin, The geometry of three-forms in six and seven dimensions, math/0010054 [INSPIRE].

[7] N.J. Hitchin, Stable forms and special metrics, math/0107101 [INSPIRE].

[8] N. Hitchin, Generalized Calabi-Yau manifolds, Quart. J. Math. Oxford Ser. 54 (2003) 281 [math/0209099] [INSPIRE].

[9] M. Gualtieri, Generalized complex geometry, math/0401221 [INSPIRE].

[10] C.M. Hull, Generalised Geometry for M-theory, JHEP 07 (2007) 079 [hep-th/0701203] [INSPIRE].

[11] P.P. Pacheco and D. Waldram, M-theory, exceptional generalised geometry and superpotentials, JHEP 09 (2008) 123 [arXiv:0804.1362] [INSPIRE].

[12] M. Graña, R. Minasian, M. Petrini and A. Tomasiello, Generalized structures of $N=1$ vacua, JHEP 11 (2005) 020 [hep-th/0505212] [INSPIRE].

[13] C. Jeschek and F. Witt, Generalised $G_{2}$ - structures and type IIB superstrings, JHEP 03 (2005) 053 [hep-th/0412280] [INSPIRE].

[14] M. Graña, J. Louis and D. Waldram, Hitchin functionals in $N=2$ supergravity, JHEP 01 (2006) 008 [hep-th/0505264] [INSPIRE].

[15] M. Graña, J. Louis and D. Waldram, $\mathrm{SU}(3) \times \mathrm{SU}(3)$ compactification and mirror duals of magnetic fluxes, JHEP 04 (2007) 101 [hep-th/0612237] [INSPIRE].

[16] M. Graña, J. Louis, A. Sim and D. Waldram, E7(7) formulation of $N=2$ backgrounds, JHEP 07 (2009) 104 [arXiv:0904.2333] [INSPIRE].

[17] J. Louis and A. Micu, Type 2 theories compactified on Calabi-Yau threefolds in the presence of background fluxes, Nucl. Phys. B 635 (2002) 395 [hep-th/0202168] [INSPIRE].

[18] A.-K. Kashani-Poor and R. Minasian, Towards reduction of type-II theories on $\mathrm{SU}(3)$ structure manifolds, JHEP 03 (2007) 109 [hep-th/0611106] [INSPIRE].

[19] R. D'Auria, S. Ferrara and M. Trigiante, On the supergravity formulation of mirror symmetry in generalized Calabi-Yau manifolds, Nucl. Phys. B 780 (2007) 28 [hep-th/0701247] [INSPIRE].

[20] P. Koerber and L. Martucci, From ten to four and back again: How to generalize the geometry, JHEP 08 (2007) 059 [arXiv:0707.1038] [INSPIRE].

[21] D. Cassani and A. Bilal, Effective actions and $N=1$ vacuum conditions from $\mathrm{SU}(3) \times \mathrm{SU}(3)$ compactifications, JHEP 09 (2007) 076 [arXiv:0707.3125] [INSPIRE].

[22] D. Cassani, Reducing democratic type-II supergravity on $\mathrm{SU}(3) \times \mathrm{SU}(3)$ structures, JHEP 06 (2008) 027 [arXiv: 0804.0595] [INSPIRE].

[23] D. Cassani and A.-K. Kashani-Poor, Exploiting $N=2$ in consistent coset reductions of type IIA, Nucl. Phys. B 817 (2009) 25 [arXiv:0901.4251] [INSPIRE]. 
[24] A. Strominger, Yukawa Couplings in Superstring Compactification, Phys. Rev. Lett. 55 (1985) 2547 [INSPIRE].

[25] A. Strominger, special geometry, Commun. Math. Phys. 133 (1990) 163 [InSPIRE].

[26] P. Candelas and X. de la Ossa, Moduli Space of Calabi-Yau Manifolds, Nucl. Phys. B 355 (1991) 455 [INSPIRE].

[27] A. Strominger, Loop corrections to the universal hypermultiplet, Phys. Lett. B 421 (1998) 139 [hep-th/9706195] [INSPIRE].

[28] I. Antoniadis, S. Ferrara, R. Minasian and K.S. Narain, $R^{4}$ couplings in $M$ and type-II theories on Calabi-Yau spaces, Nucl. Phys. B 507 (1997) 571 [hep-th/9707013] [INSPIRE].

[29] H. Gunther, C. Herrmann and J. Louis, Quantum corrections in the hypermultiplet moduli space, Fortsch. Phys. 48 (2000) 119 [hep-th/9901137] [InSPIRE].

[30] K. Becker, M. Becker, M. Haack and J. Louis, Supersymmetry breaking and alpha-prime corrections to flux induced potentials, JHEP 06 (2002) 060 [hep-th/0204254] [INSPIRE].

[31] I. Antoniadis, R. Minasian, S. Theisen and P. Vanhove, String loop corrections to the universal hypermultiplet, Class. Quant. Grav. 20 (2003) 5079 [hep-th/0307268] [INSPIRE].

[32] D. Robles-Llana, F. Saueressig and S. Vandoren, String loop corrected hypermultiplet moduli spaces, JHEP 03 (2006) 081 [hep-th/0602164] [INSPIRE].

[33] L. Andrianopoli, M. Bertolini, A. Ceresole, R. D'Auria, S. Ferrara et al., N=2 supergravity and $N=2$ super Yang-Mills theory on general scalar manifolds: Symplectic covariance, gaugings and the momentum map, J. Geom. Phys. 23 (1997) 111 [hep-th/9605032] [INSPIRE].

[34] B. de Wit and A. Van Proeyen, Potentials and Symmetries of General Gauged N=2 Supergravity: Yang-Mills Models, Nucl. Phys. B 245 (1984) 89 [inSPIRE].

[35] B. Craps, F. Roose, W. Troost and A. Van Proeyen, What is special Kähler geometry?, Nucl. Phys. B 503 (1997) 565 [hep-th/9703082] [INSPIRE].

[36] J. Bagger and E. Witten, Matter Couplings in $N=2$ Supergravity, Nucl. Phys. B 222 (1983) 1 [INSPIRE].

[37] B. de Wit, P.G. Lauwers and A. Van Proeyen, Lagrangians of $N=2$ Supergravity - Matter Systems, Nucl. Phys. B 255 (1985) 569 [inSPIRE].

[38] S. Cecotti, S. Ferrara and L. Girardello, Geometry of Type II Superstrings and the Moduli of Superconformal Field Theories, Int. J. Mod. Phys. A 4 (1989) 2475 [InSPIRE].

[39] S. Ferrara and S. Sabharwal, Dimensional Reduction of Type II Superstrings, Class. Quant. Grav. 6 (1989) L77 [INSPIRE].

[40] S. Ferrara and S. Sabharwal, Quaternionic Manifolds for Type II Superstring Vacua of Calabi-Yau Spaces, Nucl. Phys. B 332 (1990) 317 [inSPIRE].

[41] A. Strominger, Massless black holes and conifolds in string theory, Nucl. Phys. B 451 (1995) 96 [hep-th/9504090] [INSPIRE].

[42] M.B. Green, M. Gutperle and P. Vanhove, One loop in eleven-dimensions, Phys. Lett. B 409 (1997) 177 [hep-th/9706175] [INSPIRE].

[43] J.T. Liu and R. Minasian, Higher-derivative couplings in string theory: dualities and the B-field, arXiv:1304.3137 [INSPIRE]. 
[44] K.-P. Gemmer, Orientifolds and $R^{4}$-couplings on generalized geometries, Hamburg University diploma thesis, 2010, www.desy.de/uni-th/stringth/Works/Gemmer_Diplomarbeit.pdf.

[45] A.-K. Kashani-Poor, R. Minasian and H. Triendl, Enhanced supersymmetry from vanishing Euler number, JHEP 04 (2013) 058 [arXiv:1301.5031] [INSPIRE].

[46] A.-K. Kashani-Poor and A. Tomasiello, A stringy test of flux-induced isometry gauging, Nucl. Phys. B 728 (2005) 135 [hep-th/0505208] [INSPIRE].

[47] B. de Wit, B. Kleijn and S. Vandoren, Superconformal hypermultiplets, Nucl. Phys. B 568 (2000) 475 [hep-th/9909228] [INSPIRE].

[48] B. de Wit, M. Roček and S. Vandoren, Hypermultiplets, hyperKähler cones and quaternion Kähler geometry, JHEP 02 (2001) 039 [hep-th/0101161] [INSPIRE].

[49] B. de Wit, M. Roček and S. Vandoren, Gauging isometries on hyperKähler cones and quaternion Kähler manifolds, Phys. Lett. B 511 (2001) 302 [hep-th/0104215] [INSPIRE].

[50] R. Bohm, H. Gunther, C. Herrmann and J. Louis, Compactification of type IIB string theory on Calabi-Yau threefolds, Nucl. Phys. B 569 (2000) 229 [hep-th/9908007] [INSPIRE].

[51] U. Theis and S. Vandoren, N=2 supersymmetric scalar tensor couplings, JHEP 04 (2003) 042 [hep-th/0303048] [INSPIRE]. 